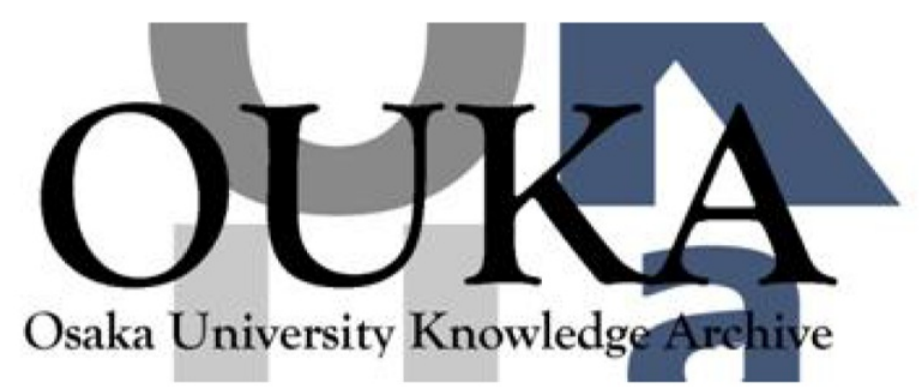

\begin{tabular}{|c|l|}
\hline Title & $\begin{array}{l}\text { Smectic layer rotation by dc field in } \\
\text { ferroelectric liquid crystal }\end{array}$ \\
\hline Author(s) & Nakayama, K.; Ozaki, M.; Yoshino, K. \\
\hline Citation & Applied Physics Letters. 70(16) p. 2117-p. 2119 \\
\hline Issue Date & $1997-04-21$ \\
\hline oaire:version & VoR \\
\hline URL & https://hdl. handle. net/11094/75844 \\
\hline rights & \\
\hline Note & \\
\hline
\end{tabular}

Osaka University Knowledge Archive : OUKA

https://ir. Library. osaka-u. ac. jp/

Osaka University 


\section{Smectic layer rotation by dc field in ferroelectric liquid crystal}

Cite as: Appl. Phys. Lett. 70, 2117 (1997); https://doi.org/10.1063/1.118966

Submitted: 02 January 1997 . Accepted: 18 February 1997. Published Online: 04 June 1998

K. Nakayama, M. Ozaki, and K. Yoshino

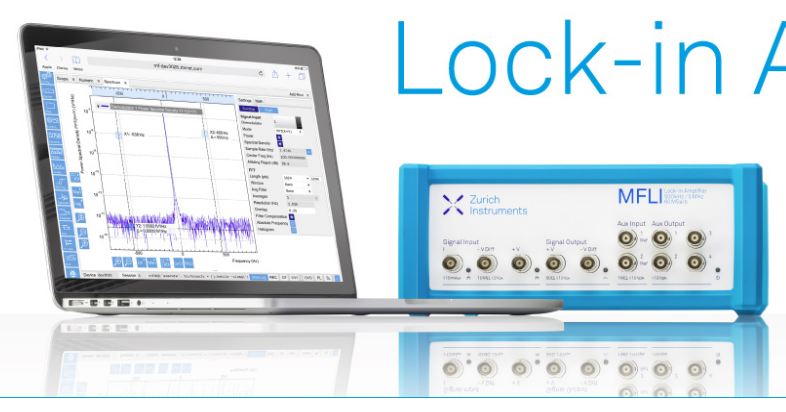

Amplifiers

Zurich

Instruments

Watch the Video 


\title{
Smectic layer rotation by dc field in ferroelectric liquid crystal
}

\author{
K. Nakayama, M. Ozaki, and K. Yoshino \\ Department of Electronic Engineering, Faculty of Engineering, Osaka University 2-1 Yamada-Oka, Suita, \\ Osaka 565, Japan
}

(Received 2 January 1997; accepted for publication 18 February 1997)

\begin{abstract}
The smectic layer rotation of a ferroelectric liquid crystal (FLC) has been investigated by applying a dc electric field. The smectic layer of FLC doped with a small amount of an ionic impurity can be rotated around the axis perpendicular to the glass plates upon application of dc voltage. This smectic layer rotation is interpreted in terms of the electrohydrodynamic flow due to an ionic impurity.

(C) 1997 American Institute of Physics. [S0003-6951(97)02716-2]
\end{abstract}

Electro-optic effects in smectic liquid crystals, especially, in chiral smectic structural ferroelectric liquid crystals (FLCs) have attracted much interest from both fundamental and practical viewpoints. In such cases, the dynamic response of molecular reorientation upon application of voltage has been discussed in the framework of a stable smectic layer structure. That is, a smectic layer structure has been believed to be not influenced by an applied field. Meanwhile, Patel and Goodby ${ }^{1}$ have previously reported the layer reorientation upon application of an electric field in ferroelectric liquid crystals. However, in this case a smectic layer tilts away from a rubbing axis by an angle equal to a molecular tilt angle and this reorientation is irreversible. Myojin et al. ${ }^{2}$ have reported the alignment control of FLC with a chiral nematic $\left(\mathrm{N}^{*}\right)$-chiral smectic $\mathrm{C}\left(\mathrm{SmC}^{*}\right)$ phase transition upon application of an ac electric field in the cell in which both substrate surfaces have been rubbed. This reorientation is also irreversible.

On the contrary, we have reported that the reversible rotation of a smectic layer by an angle much larger than a tilt angle is achieved by applying an ac electric field in FLCs and antiferroelectric liquid crystals (AFLCs). ${ }^{3-6}$ This layer rotation requires the ac electric field of an asymmetry waveform such as a sawtooth waveform, for example. This layer rotation has been considered to be unique for the ferroelectric phase and the antiferroelectric phase. However, recently we found the smectic layer rotation in the smectic A phase. ${ }^{7,8}$ A layer rotates by applying asymmetric pulses over the fieldinduced tilt angle due to the electroclinic effect.

These reversible layer rotations have been interpreted to be originated in asymmetry of molecular switching dynamics upon applying asymmetric pulses. In this letter, we report that a smectic layer rotation was also induced by applying a dc electric field in FLC doped with an ionic impurity.

The ferroelectric liquid crystal used in this study is CS1024 (Chisso Co.). The phase sequence is the isotropic (Iso) phase $\rightarrow$ the chiral nematic $\left(\mathrm{N}^{*}\right)$ phase $\rightarrow$ the smectic A (SmA) phase $\rightarrow$ the chiral smectic $\mathrm{C}\left(\mathrm{SmC}^{*}\right)$ phase. Tetracyanoquinodimethane (TCNQ) was doped as an ionic impurity into the liquid crystal with $0.5 \mathrm{wt} \%$ concentration. The sample was sandwiched between indium-tin-oxide (ITO)-coated glass plates. The glass plates were fixed using polyethyleneterephthalate (PET) as spacers and the cell gap was $6 \mu \mathrm{m}$. In the cell used in this study, the surface stabilization of the molecules was not realized and memory effect was not observed. We shall use only cells whose gap is $6 \mu \mathrm{m}$ in this letter, but similar results should be observed under another cell gap. A unidirectionally aligned monodomain was obtained by gradual growth of SmA domain from a spacer edge with the help of a thermal gradient. Experiments were carried out $5{ }^{\circ} \mathrm{C}$ below the $\mathrm{SmA}-\mathrm{SmC}^{*}$ phase transition temperature.
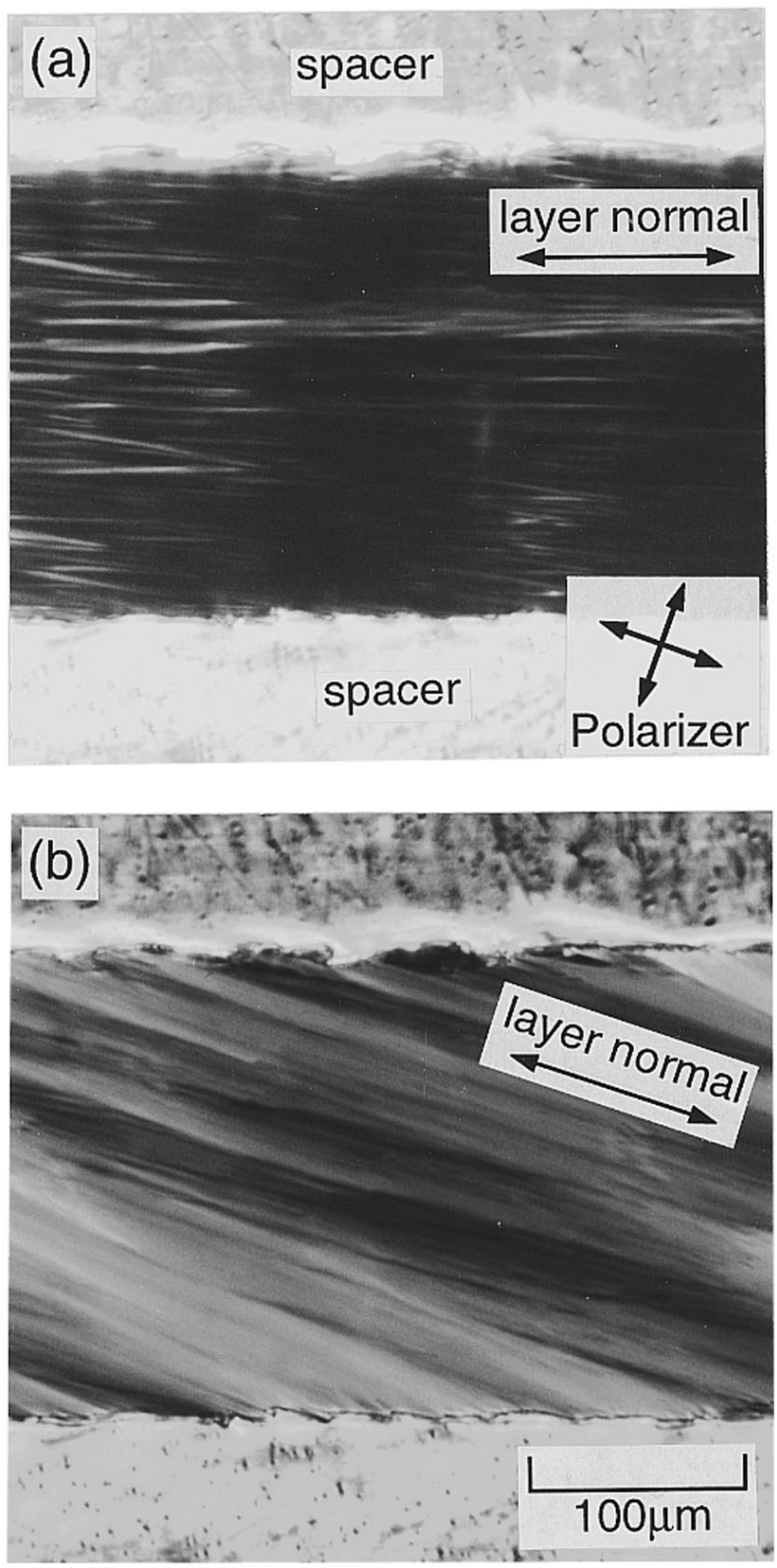

FIG. 1. Polarizing micrographs of FLC doped with $0.5 \mathrm{wt} \%$ TCNQ: (a) the initial state right after applying dc $+20 \mathrm{~V}$, (b) $10 \mathrm{~min}$ after. 


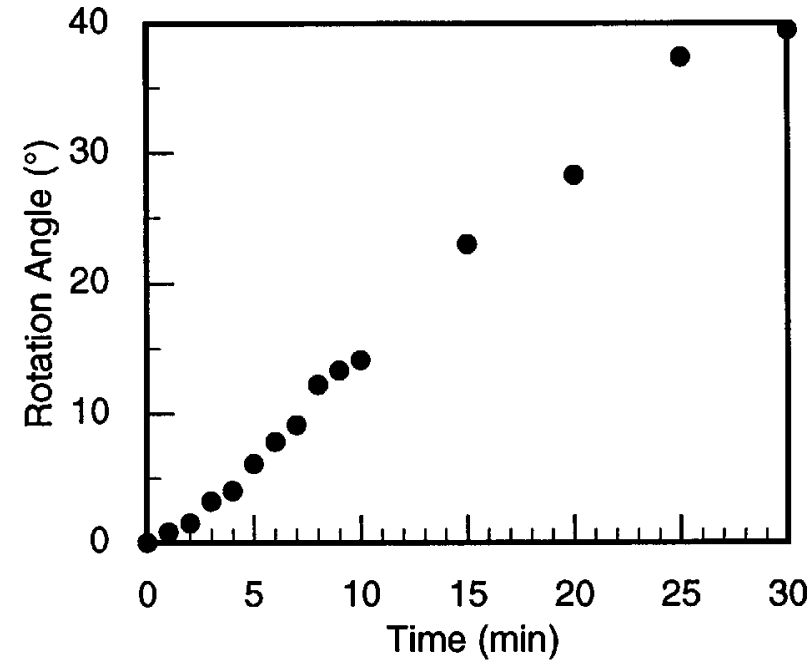

FIG. 2. A rotation angle of FLC doped with $0.5 \mathrm{wt} \%$ TCNQ as a function of the time of applying dc voltage.

The positive direction of a smectic layer rotation was defined as clockwise when positive voltage was applied to the lower electrode. The rotation angle was defined as a difference between the initial extinction direction right after applying $\mathrm{dc}$ $20 \mathrm{~V}$ and the extinction direction for a certain time.

Upon application of dc voltage $(+20 \mathrm{~V})$ on pure FLC, any remarkable changes of smectic layer structure were not observed on a polarized microscope observation. In the case of TCNQ doped sample, however, remarkable changes appeared. The series of polarizing micrographs of FLC (CS1024) doped with $0.5 \mathrm{wt} \%$ TCNQ is shown in Fig. 1. Figure 1(a) was taken at the initial state, that is, right after applying $\mathrm{dc}+20 \mathrm{~V}$ and (b) was taken $10 \mathrm{~min}$ later. In Fig. 1(a), the sample cell was set as the optical axis of the sample is parallel to the polarizer, therefore the sample looks dark except for spacers (top and bottom edges in micrographs). A smectic layer normal in Fig. 1(a) is parallel to the spacer edges.

When dc voltage of $+20 \mathrm{~V}$ was applied, however, the smectic layer began to rotate around an axis perpendicular to the sample cell and the layer rotated clockwise by about $14^{\circ}$ for $10 \mathrm{~min}$. During the rotation, a unidirectional alignment was kept [Fig. 1(b)]. Although the geometry between the cell and the polarizers in Fig. 1(b) is the same as that in Fig. 1(a), the sample looks brighter in Fig. 1(b). These figures clearly indicate that the optical axis rotated. A direction of a layer normal was determined by the observation of extinction directions at positive and negative voltage applications.

Figure 2 shows the rotation angle of FLC (CS-1024) doped with $0.5 \mathrm{wt} \%$ TCNQ as a function of the time of dc voltage application. The smectic layer began to rotate right after applying dc voltage and the rotation angle increased with time at almost constant rate. In this case, the layer rotated at least exceeding $40^{\circ}$ by application of dc voltage for $30 \mathrm{~min}$.

Moreover, the direction of the layer rotation depends on the polarity of applied dc voltage. In other words, the smectic layer rotates counterclockwise when negative dc voltage is applied. It should also be mentioned that this layer rotation is reversible. In this sample, the smectic layer rotated in the
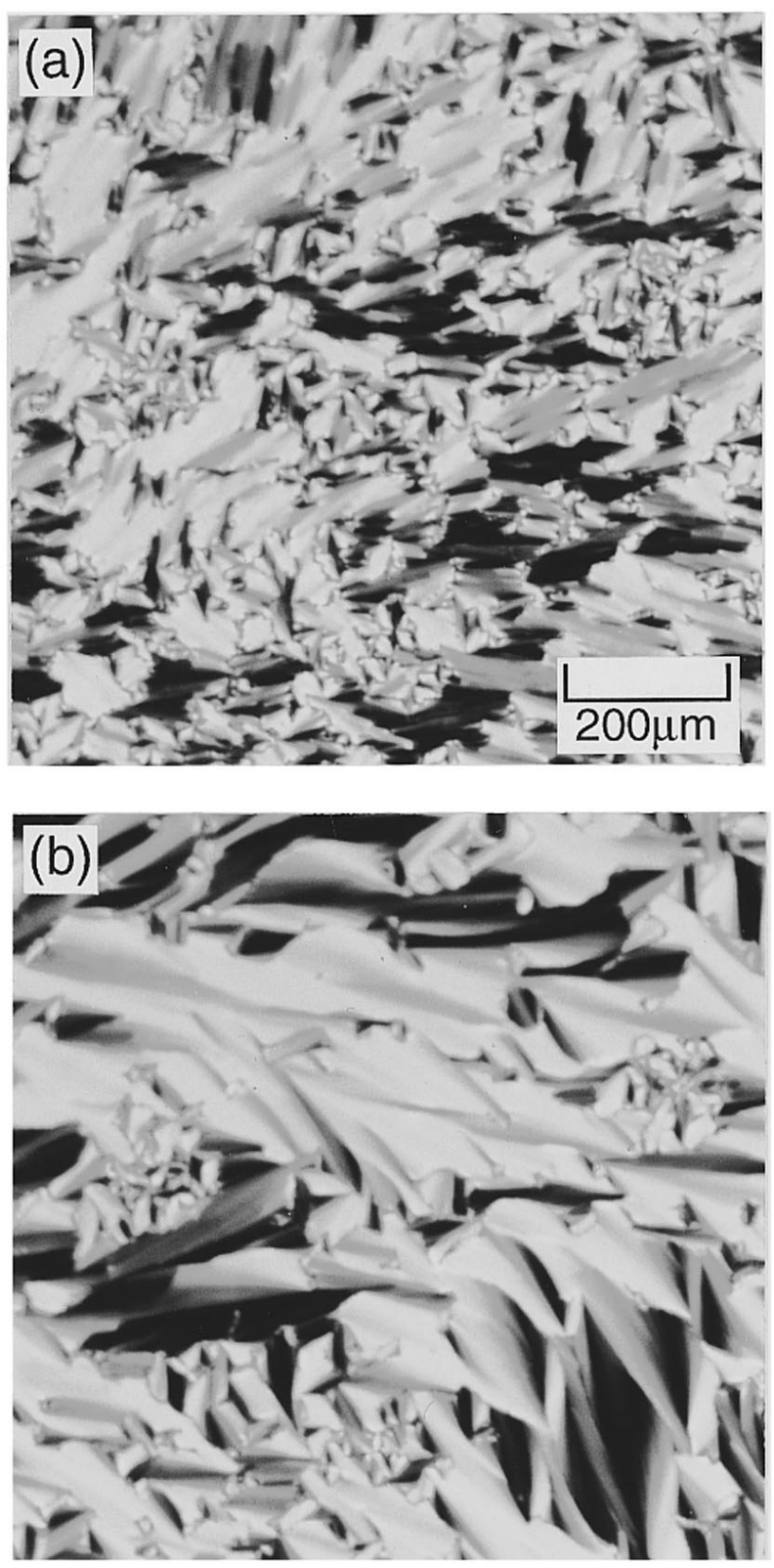

FIG. 3. Polarizing micrographs of FLC doped with 0.5 wt $\%$ TCNQ in the multidomain cell: (a) the initial state, (b) 90 min after applying dc $+20 \mathrm{~V}$.

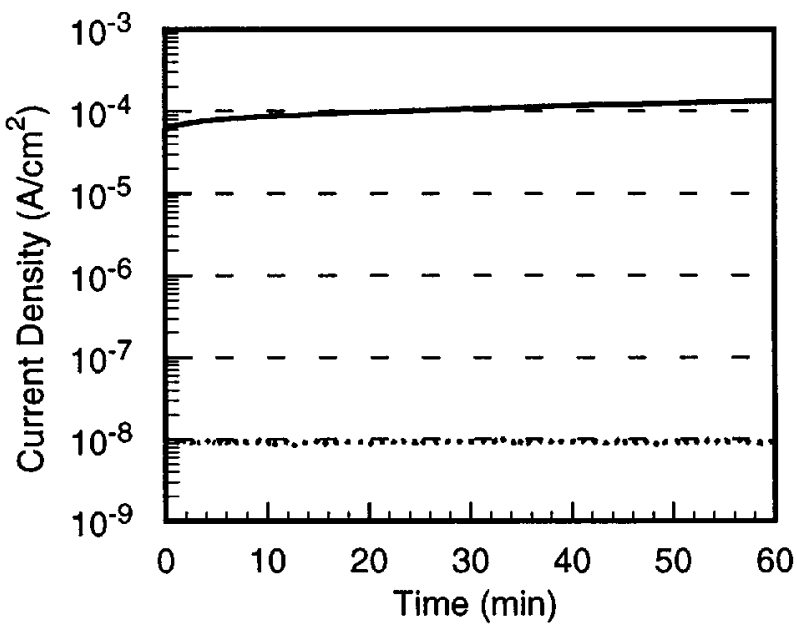

FIG. 4. The current density under application of dc $+20 \mathrm{~V}$. The cell gap was $6 \mu \mathrm{m}$ and the area is $5 \times 5 \mathrm{~mm}^{2}$. Dotted line: pure sample, Solid line: sample doped with 0.5 wt \% TCNQ. 
same direction to the molecular tilt under application of $\mathrm{dc}$ voltage.

This layer rotation was also observed in a multidomain cell of a doped sample. The cell used for this experiment was not treated for unidirectional alignment and the thermal gradient method was not used. Figure 3(a) shows a polarizing micrograph at the initial state before field application. Figure 3(b) was a micrograph taken 90 min after applying dc $+20 \mathrm{~V}$. The number of defects decreased and the border of domains became unnoticed, that is, each domain grew and neighboring domains were fused with each other when applying dc voltage. The layer in most domains rotates in the same direction which is determined by the molecular tilt direction, as mentioned above. However, it should also be noted that there are some domains in which the layer rotates in the opposite direction even upon dc field application of the same polarity. Namely, domains with an opposite rotation coexist in a multidomain cell. For a tentative explanation of this anomaly, it may be necessary to take into account the interaction between neighboring domains, since such phenomenon was observed only in a multidomain cell.

A pure sample without any ionic impurities did not show the layer rotation by applying dc voltage. In other words, the layer rotation by de voltage occurs only in the FLC sample in which a resistivity was reduced by doping with an ionic impurity. To confirm the importance of the reduction of a resistance for the layer rotation, we measured a current through the sample under application of dc voltage. The current density through the pure sample under dc $20 \mathrm{~V}$ was about $\mathrm{nA} / \mathrm{cm}^{2}$ and constant (the dotted line in Fig. 4). While, the current density in the TCNQ doped sample (0.5 wt \%) was about $100 \mu \mathrm{A} / \mathrm{cm}^{2}$ (the solid line in Fig. 4), which is about 1000 times as large as that in the pure sample. Moreover, the current was not constant but slightly increasing. These results indicate that the current plays an important role in the layer rotation by applying dc voltage. In fact, the perturbation of a texture is observed with a polarizing microscope during application of dc voltage, which would be caused by a flow of ions, although the smectic layer structure is conserved. Namely, the layer rotation upon dc voltage is associated with the electrohydrodynamic flow in the smectic layer.

The smectic layer rotation in FLCs and AFLCs, which has been reported by us, requires an asymmetric polarization reversal of applied voltage. ${ }^{3-8}$ For example, a sawtooth waveform can induce the layer rotation. Namely, these layer rotations should be originated in the asymmetry of driving force due to molecular switching achieved by applying asymmetric pulses. Moreover, the layer rotation upon asymmetric ac voltage can be realized even in a pure sample. On the other hand, the layer rotation observed in this study takes place only upon application of dc voltage and need neither asymmetry of the voltage waveform nor the polarization reversal. In addition, this novel layer rotation was accompanied by the electrohydrodynamic flow in the smectic layer. For the pure sample, the FLC molecules keep tilting away from the layer normal and do not move under application of the dc field. Therefore, no dynamic phenomena should happen in this system. For the ionic impurity doped FLC, however, although the molecules also tilt away from the layer normal, the ionic impurity move by electric field, which causes the flow of impurity molecules as mentioned above. That is, in the case of the doped sample, the violent fluctuation of the FLC molecules should be induced, which might cause interlayer movement of the FLC molecules and perturbation of the layer alignment by the flow. Consequently, this rearrangement of the smectic layer may result in the layer reorientation. Although the origin of rearrangement of the smectic layer of the dc-field-induced layer rotation might be different from that of the ac-field-induced layer rotation reported so far, ${ }^{3-6}$ violent molecular motion which destroys the layer structure for an instant should arise in both cases.

In conclusion, the smectic layer of FLC doped with an ionic impurity rotates under application of dc voltage. In addition, the rotation is reversible and the direction of the layer rotation depends on the polarity of applied dc voltage. The possibility of realignment of the smectic layer influenced by application of dc voltage is important not only from a fundamental but also from a practical point of view.

A part of this work was supported by Grant-in-Aid for General Scientific Research from the Ministry of Education, Science, Sports and Culture.

${ }^{1}$ J. S. Patel and J. W. Goodby, J. Appl. Phys. 59, 2355 (1986).

${ }^{2}$ K. Myojin, H. Moritake, M. Ozaki, K. Yoshino, T. Tani, and K. Fujisawa, Jpn. J. Appl. Phys. 33, 5491 (1994).

${ }^{3}$ M. Ozaki, H. Moritake, K. Nakayama, and K. Yoshino, Jpn. J. Appl. Phys. 33, L1620 (1994)

${ }^{4}$ H. Moritake, K. Nakayama, M. Ozaki, and K. Yoshino, Mol. Cryst. Liq. Cryst. 263, 13 (1995).

${ }^{5}$ K. Nakayama, H. Moritake, M. Ozaki, and K. Yoshino, Jpn. J. Appl. Phys. 34, L1599 (1995)

${ }^{6}$ H. Moritake, K. Nakayama, M. Ozaki, and K. Yoshino, Ferroelectrics 178, 277 (1996).

${ }^{7}$ K. Nakayama, M. Ozaki, and K. Yoshino (to be published in Mol. Cryst. Liq. Cryst.).

${ }^{8}$ K. Nakayama, M. Ozaki, and K. Yoshino, Jpn. J. Appl. Phys. 35, 6200 (1996). 